\title{
СИНТЕЗ НАБЛЮДАТЕЛЯ ДЛЯ БИЛИНЕИНЫХ СИСТЕМ С ДИСКРЕТНЫМ ВРЕМЕНЕМ
}

Olle KOTTA. OLEKU TAASTAJA KONSTRUEERIMINE DISKREETSE AJAGA BILINEAARSETE SUSTEEMIDE JAOKS

OIle KOTTA. OBSERVER DESIGN FOR DISCRETE TIME BILINEAR SYSTEMS

\author{
(Представил Н. Алумяэ)
}

1. Введение. Задача синтеза наблюдателя для билинейных систем привлекает внимание многих авторов $\left[{ }^{1-10}\right]$. Основная трудность при решении ее заключается в том, что надо обеспечить асимптотическую устойчивость системы, описывающей поведение ошибки восстановления, в то время как динамика ошибки зависит от управления. Для этого существуют два приема. Первый требует, чтобы наблюдаемая система удовлетворяла неким сильным структурным ограничениям, которые обеспечивают описание динамики ошибки линейной, устойчивой, стационарной системой [ $\left.{ }^{1}\right]$. Другой способ можно применять при любых билинейных системах, но при этом требуется ограничить пространство допустимых управлений. Рассматривались случаи равномерно ограниченных входов $\left[{ }^{2-5}\right]$ и экспоненциально ограниченных входов [ $\left.{ }^{6}\right]$. В последнем случае синтез наблюдателя особенно прост. Во всех этих работах рассматривались системы с непрерывным временем. Целью нашей работы было распространение результатов работы $\left[{ }^{6}\right]$ на системы с дискретным временем.

2. Основной результат. Рассмотрим билинейную систему с дискретным временем

$$
\begin{gathered}
x(t+1)=A x(t)+\sum_{i=1}^{m} B_{i} x(t) u_{i}(t)+C u(t), \\
y(t)=D x(t),
\end{gathered}
$$

где $x \in R^{n}, u=\left[u_{1}, \ldots, u_{m}\right] \in R^{m}, y \in R^{p}(p<n)$, а матрицы $A, B_{1}, \ldots$ $\ldots, B_{m}, C$ и $D$ подходящей размерности. Начальное состояние $x(0)$ неизвестно, входы $u(t)$ и выходы $y(t)$ при $t=1,2, \ldots$ известны. Предположим, что входы являются экспоненциально устойчивыми

$$
\left|u_{i}(t)\right| \leqslant r_{1} r_{2}^{t}, \quad r_{1}>0, \quad r_{2}>1, \quad i=1, \ldots, m .
$$

Ставится задача восстановить состояние $x(t)$ системы (1) по наблюдаемым переменным $u(t)$ и $y(t)$. Для решения подобных задач обычно вводят другую динамическую систему, т. н. наблюдатель

$$
\begin{aligned}
z(t+1) & =A z(t)+\sum_{i=1}^{m} B_{i} z(t) u_{i}(t)+C u(t)+ \\
& +G[u(t)]\{y(t)-D z(t)\} \\
& G[u(t)]=G_{0}+\sum_{i=1}^{m} G_{i} u_{i}(t)
\end{aligned}
$$


состояние $z(t)$ которой должно являться апгіроксимацией состояния $x(t)$ в определенном смысле.

Из уравнений (1) и (2) следует, что ошибка восстановления $e(t)=$ $=x(t)-z(t)$ удовлетворяет билинейному уравнению

$$
e(t+1)=\left[\widetilde{A}+\sum_{i=1}^{m} \widetilde{B}_{i} u_{i}(t)\right] e(t)
$$

где $\tilde{A}=A-G_{0} D$ и $\widetilde{B}_{i}=B_{i}-G_{i} D$.

Оп р еделенн е. Билинейная система (3) экспоненциально устойчива, если существуют такие $\mu>0$ и $\lambda>1$, что все решения системы (3) обладают свойством

$$
\|e(t)\| \leqslant \mu \lambda^{-t}\|e(0)\|
$$

при любых $t=1,2, \ldots, e(0) \in R^{n} u$ допустимых $u($.$) .$

Докажем следующую теорему.

Теорем а. Билинейная система (3) является при экспоненциально ограниченных входах экспоненциально устойчивой, если выполнены следующие условия:

1) $\left\|\tilde{A}^{t}\right\| \leqslant s_{1} s_{2}^{-t}, \quad s_{1}>0, \quad s_{2}>1$,

2) $r_{1}<\left(1-\frac{1}{r_{2}}\right) \frac{1}{M m s_{1} s_{2}}$, где $M=\max _{i=1, \ldots, m}\left\|\widetilde{B}_{i}\right\|$.

Доказательство. Выпишем решение уравнения (3)

$$
\begin{aligned}
e(t)= & \widetilde{A}^{t} e(0)+\sum_{i_{1}=1}^{m} \sum_{k_{1}=0}^{t-1} \widetilde{A}^{t-k_{1}-1} \widetilde{B}_{i_{1}} \widetilde{A}^{k_{1}} e(0) u_{i_{1}}\left(k_{1}\right)+ \\
+ & \sum_{i_{1}, i_{2}=1}^{m} \sum_{k_{1}=0}^{t-2} \sum_{k_{2}=k_{1}+1}^{t-1} \widetilde{A}^{t-k_{2}-1} \widetilde{B}_{i_{2}} A^{k_{2}-k_{1}-1} \widetilde{B}_{i_{1}} \widetilde{A}^{k_{1}} \times \\
& \times e(0) u_{i_{1}}\left(k_{1}\right) u_{i_{2}}\left(k_{2}\right)+\ldots \\
& \ldots+\sum_{i_{1}, \ldots, i_{l}=1}^{m} \widetilde{B}_{i_{1}} \ldots \widetilde{B}_{i_{t}} e(0) u_{i_{1}}(0) \ldots u_{i_{t}}(t-1)
\end{aligned}
$$

Воспользуясь свойствами нормы

$$
\begin{gathered}
\|A+B\| \leqslant\|A\|+\|B\|, \\
\|A B\| \leqslant\|A\|\|B\|, \\
\|c A\|=|c|\|A\|,
\end{gathered}
$$

получим

$$
\begin{aligned}
\|e(t)\| & \leqslant s_{1} s_{2}^{-t}\|e(0)\|+m \sum_{k_{1}=0}^{t-1} s_{1} S_{2}^{-t+k_{1}+1} M s_{1} s_{2}^{-k_{1}} r_{1} r_{2}^{-k_{1}}\|e(0)\|+ \\
& +m^{2} \sum_{k_{1}=0}^{t-2} \sum_{k_{2}=k_{1}+1}^{t-1} s_{1} s_{2}^{-t+k_{2}+1} M s_{1} s_{2}^{-k_{2}+k_{1}+1} M s_{1} s_{2}^{-k_{1}}\|e(0)\| r_{1} r_{2}^{-k_{2}} r_{1} r_{2}^{-k_{1}}+\ldots \leqslant \\
& \leqslant s_{1} s_{2}^{-t}\|e(0)\|\left\{1+s_{1} s_{2} m M r_{1} \sum_{k_{1}=0}^{t-1} r_{2}^{k_{1}}+\right. \\
& \left.+s_{1}^{2} s_{2}^{2} m^{2} M^{2} r_{1}^{2} \sum_{k_{1}=0}^{t-2} r_{2}^{-k_{1}} \sum_{k_{2}=k_{1}+1}^{t-1} r_{2}^{-k_{2}}+\ldots\right\} \leqslant
\end{aligned}
$$




$$
\begin{aligned}
& \leqslant s_{1} s_{2}{ }^{t}\|e(0)\|\left\{1+s_{1} s_{2} m M r_{1} \frac{r_{2}}{r_{2}-1}+s_{1}^{2} s_{2}^{2} m^{2} M^{2} r_{1}^{2}\left(\frac{r_{2}}{r^{2}-1}\right)^{2}+\ldots\right\} \leqslant \\
& \leqslant \frac{s_{1} s_{2}^{-t}}{1-\mu_{1}}\|e(0)\| ;
\end{aligned}
$$

где $\mu_{1}=s_{1} s_{2} m M r_{1} \frac{r_{2}}{r_{2}-1}$.

Теорема доказана.

Из теоремы следует, что для решения поставленной задачи мы должны выбрать в уравнениях наблюдателя (2) $G_{0}$ таким образом, чтобы все собственные числа матрицы $A-G_{0} D$ были расположены внутри единичного круга. Это гарантирует выполнение первого условия теоремы.

Из теории линейных систем хорошо известно, что этого можно добиться, если $(A, D)$ наблюдаемая пара. Выбор матриц $G_{i}, i=1, \ldots, m$ и начального состояния наблюдателя $z(0)$ произвольный.

3. Пример. Рассмотрим систему

$$
\begin{gathered}
x(t+1)=\left[\begin{array}{rr}
2,2 & -0,1 \\
10 & 0,2
\end{array}\right] x(t)+\left[\begin{array}{cc}
1 & 0 \\
0,4 & 1
\end{array}\right] x(t) u(t)+\left[\begin{array}{l}
1 \\
2
\end{array}\right] u(t), \\
y(t)=\left[\begin{array}{ll}
1 & 0
\end{array}\right] x(t) .
\end{gathered}
$$

Если мы выберем в уравнениях наблюдателя $\quad G_{0}=\left[\begin{array}{c}2 \\ 10,6\end{array}\right]$, то $\tilde{A}=A-G_{0} D=\left[\begin{array}{rr}0,2 & -0,1 \\ -0,6 & 0,2\end{array}\right]$ удовлетворяет условиям теоремы. Мы можем взять, например, $s_{2}=2$ и $s_{1}=1,6$. Выбор $G_{1}$ произвольный. Выберем $G_{1}=0$. При таком выборе $G_{1}, s_{1}$ и $s_{2}$, величины $m_{1}$ и $m_{2}$ должны удовлетворять следующему неравенству $m_{1}<5\left(m_{2}-1\right) / 1,4 \cdot 2 \cdot 8$.

\section{ЛИ ТЕ РА Т У Р А}

1. Hara, S., Furuta, K. Int. J. Contr., 24, № 5, 705-718 (1976).

2. Derese, I., Stevens, P., Noldus, E. Int. J. Syst. Sci., 10, № 6, 649-668 (1979)

3. Derese, I. A., Noldus, E. J. IEEE Trans. Autom. Contr., 26, № 2, 590-592 (1981).

4. Adachi, Y., Funahashi, Y. Inform. Sci. 19, № 1, 67-80 (1979).

5. Adachi, Y., Funahashi, Y. Trans. Inst. Electron. and Commun. Eng., E62, № 6, 407 (1979).

6. Hsu, C. S., Karanam, V. R. Trans. ASME. J. Dyn. Systems, Meas. and Contr., 105, № 3, 206-208 (1983).

7. Williamson D. Automatica, 13, № 3, 243-254 (1977).

8. Derese, I., Stevens P., Noldus, E. Journal A, 20, № 4, 193-202 (1979).

9. Funahashi, Y., Res. Rep. Autom. Control Lab. Fac. Eng. Nagoya Univ., 126, 53-60 (1979)

10. Funahashi, Y. Res. Rep. Autom. Control Lab. Fac. Eng. Nagoya Univ., 126, 46-52 (1979).

Институт кибернетики

Академии наук Эстонской ССР
Поступила в редакцию 2/XI 1984 